\title{
Development of prosthetic arm using body actioned SEMG signals
}

\author{
Karan Veer $^{*, \S}$, Tanu Sharma ${ }^{\dagger}$ and Amod Kumar* \\ *EIED Department, Thapar University, Patiala, India \\ ${ }^{\dagger}$ CSED Department, Global College of Engineering, Khanpur Kui, India \\ *Chief Scientist, CSIO-CSIR, Chandigarh, India \\ §karan.una@gmail.com
}

Received 1 October 2015

Accepted 16 December 2015

Published 24 February 2016

\begin{abstract}
People's working capability is badly affected when they suffer an amputated arm. Artificial replacements with prosthetic devices to get a satisfactory level of performance for essential functions with the currently available prosthetic technology are very difficult. Myoelectric arm prostheses are becoming popular because they are operated by a natural contraction of intact muscles. Hence, SEMG based artificial arm was fabricated. The system consists of different electronic and mechanical assemblies for operation of hand utilizing microcontroller in order to have minimum signal loss during its processing. With the help of relay switching connected to low power DC motor, system is capable of opening and closing of grip according to individual wish.
\end{abstract}

Keywords: SEMG electrodes; variable gripping; myoelectric controls; electronic interface; signals processing.

\section{Motivation of Study}

After limb amputation, the control strategy of muscles is no longer available. Development of a natural surface electromyogram (SEMG) based control strategy based on the natural muscular activities selected by the central nervous system is still required with low cost. Study of SEMG at different locations and interpretations of different parameters is helpful for better understanding the behavior of SEMG and muscle operation. ${ }^{1,2}$ For expansion and improvement of amputee's activity of daily living, several kinds of SEMG controlled prosthetic hands are under development.

The use of SEMG to control the prosthetic hand provides the amputees with a control feeling similar to moving their normal hand. Actuators and mechanisms research area shows lot of technological advances in externally made active prostheses. The advances in sensors include the use of piezoelectric materials, special metal alloys, polymers and new motor applications while the advances in mechanisms include mechanical designs based on the anatomy of

$\S_{\text {Corresponding author. }}$

This is an Open Access article published by World Scientific Publishing Company. It is distributed under the terms of the Creative Commons Attribution 4.0 (CC-BY) License. Further distribution of this work is permitted, provided the original work is properly cited. 
the human hand and improvements in the way these components are combined.

The simplest version of artificial limb looks like the human limb called as cosmetic prosthesis. It is made up of latex or silicon but is not able to perform any activity. It is used to hide distorted image of an amputee. Another type is body powered prosthesis in which control cables are operated through shoulder's movement. In this case, specified operations are performed robustly. In a simple robotic structure of human hand, control is through switches. Usually, a small motor provides functions of hand. The next version of electronic hands is the myoelectric controlled prosthesis wherein actuation and control is done through proper sensing of movements of muscles. A SEMG detector signal from different motor units is a composite of different signals of individual muscle for further analysis for the development of prosthetic arm..$^{3-10}$

\section{Introduction}

The SEMG is the recording of muscle electrical activity and is measured by electrodes affixed to the surface of the skin, above the muscle of interest so that rich useful information can be obtained from the muscles. Surface electromyogram may be affected by various peripheral factors such as spacing, type and placement of electrodes on desired location. For example, the electrodes aligned along the muscle length close to each other results in an increase of higher frequency contents; Researchers can use such information in a wide class of clinical and engineering applications. Currently, SEMG is largely used to determine binary states, such as high/ low contraction strength or fatigued/not fatigued muscles.

The SEMG is the recorded sequences of two principal bioelectric activities:

(a) Propagation of motor nerve impulses and their transmission at the neuromuscular junctions of a motor unit, and

(b) Propagation of muscle impulses by the sarcolemma and the T-tubular systems resulting in excitation and contraction coupling.

In order to predict the effect of SEMG corresponding to voluntary muscle contraction, various models have been developed by researchers. ${ }^{11}$ With computers and software becoming more and more powerful tools to process complex algorithms on large data at high speed, SEMG features can be computed in numerical form from a finite length epoch which changes as a function of time, i.e., a voltage or a frequency. They can be computed in time domain, frequency domain, and time frequency representations, since the magnitude of the action potentials of active motor units are not all the same nor are they in phase with one another and even the timing sequence of motor unit activation is also variable which results in complex SEMG. ${ }^{12,13}$

\section{Comparison to Previous Studies}

In fact, it is difficult to compare previous results due to nonuniformity in protocols and also due to different ways of analyzing, interpreting and presenting the results. ${ }^{14}$ However, some models like Kuiken's prostheses within Defense Advanced Research projects Agency (DARPA) project, the Utah arm (with $913 \mathrm{gm}$ arm and $450 \mathrm{gm}$ of hand weight), Boston digital arm (1,020 gm weight with single dof), the Touch bionic hand, Otto bock dynamic arm (weight $50 \mathrm{gm}$ with $140 \mathrm{~N}$ of force) etc have been developed in this regard by designers. . $^{3,7,15,16}$ The device reported in this work used following techniques:

(a) Dynamic mode control system allowed both grip speed and force to be proportionally controlled by both electrodes at all opening widths. The level of the muscle signal determines the gripping speed and force resulting in a more physiological gripping function;

(b) Grip force system used two electrodes and offers two levels of gripping force control. With this system, a mild contraction of the flexor muscle closes the hand to a low force (approximately $10 \mathrm{~N}$ ) only. A stronger contraction of the same muscle builds grip force beyond this point;

\section{Methodology}

The block diagram of electronic circuit for the operation of hand is as given in Fig 1.

Description of various modules of this setup is as below:

\section{(i) Differential amplifier}

The signal obtained from the electrodes is of the order of microvolts. It is transmitted to the 


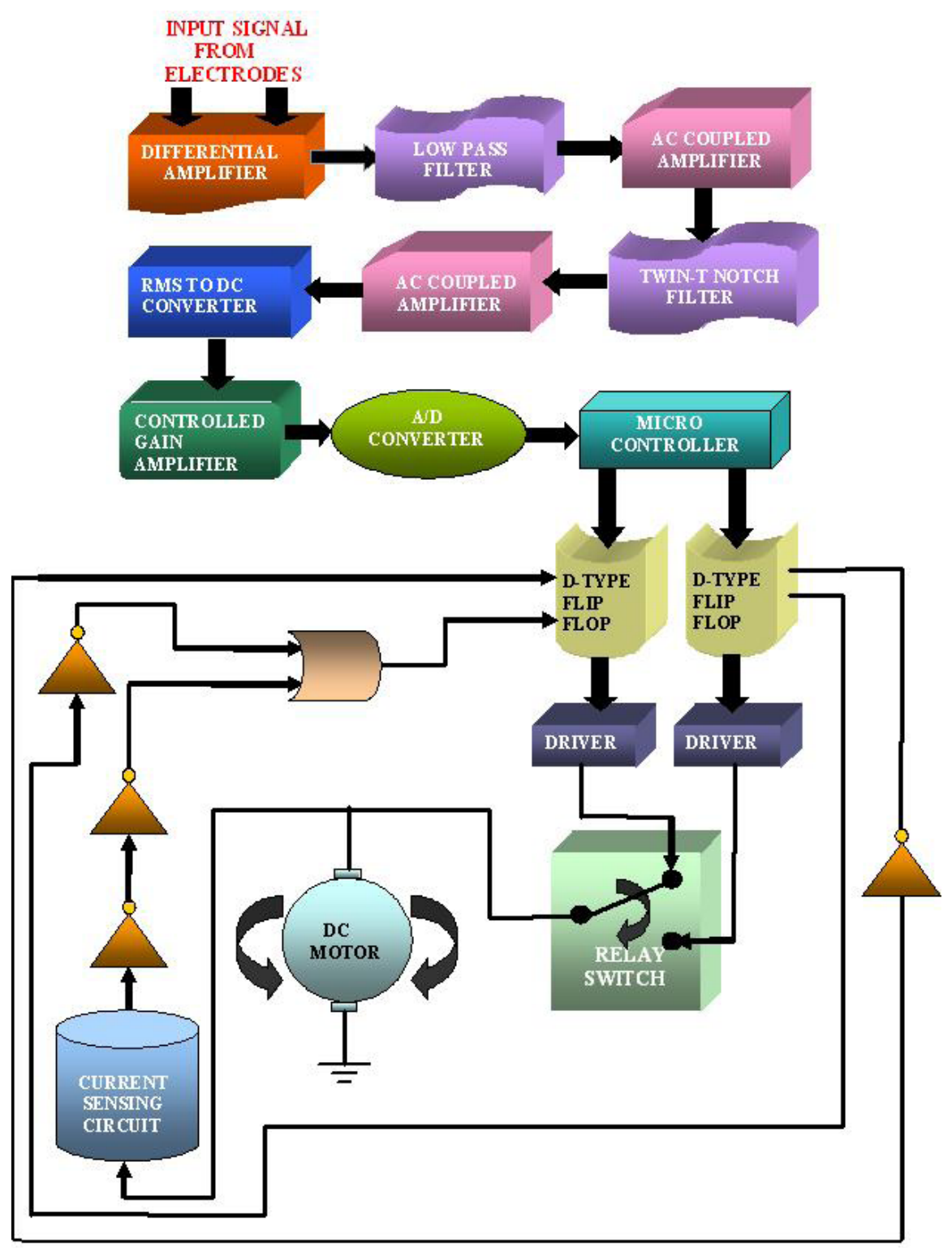

Fig. 1. Block diagram for myoelectric arm.

pre-amplification circuitry through shielded cables to reduce transmission losses.

The purpose of differential amplifier is to get the difference of muscle contraction between the two locations of the arm. The signals from both electrodes are fed to an amplifier which takes care of common mode noise. During testing, the reference voltage has been set at $3.04 \mathrm{~V}$. This configuration resolves the difference more precisely and makes the output more sensitive to the change in the state of the hand. Even a small relaxation or contraction of the hand shows a measurable variation in the SEMG signal potential.

\section{(ii) Low-pass filter}

Since the myoelectric potential used for control is so small, there can be problem from outside interferences like $50 \mathrm{~Hz}$ electrical noise. There are many sources of noise which are strong enough to operate the prosthesis erroneously. These include radio signals, static electricity etc apart from power line ripples. A filter removes all these noise signals. The passive RC lowpass filter has a cut-off frequency of about $100 \mathrm{~Hz}$.

\section{(iii) $A C$ coupled amplifier and notch filter}

This amplifier is used to increase the gain further so that signal can be properly analyzed. The gain of 
this stage is about 100. The notch filter (notch at $50 \mathrm{~Hz}$ ) eliminates any interference due to nearby power lines.

\section{(iv) $R M S$ to $D C$ converter}

This is used to convert the AC value into proportional DC value, so that the signal level may be properly converted into equivalent digital value. The AD 536 is a complete monolithic integrated circuit which performs true RMS to DC conversion. It offers performance which is comparable or superior to that of hybrid or modular units costing much more. The AD 536 directly computes the RMS value of any complex input waveform containing $\mathrm{AC}$ and $\mathrm{DC}$ components.

\section{(v) Analog to digital converter}

This analog signal is converted into digital number by an 8-bit A/D converter so that the signal level may be fed to microcontroller to analyze it.

\section{(vi) Microcontroller}

The 20 pin microcontroller $89 \mathrm{C} 2051$ is used here in view of less space available. It controls all the operation sequences like motor operations, controlling relay switching matrix etc.

\section{Gripping Force Protocol}

The developed myoelectric arm is similar to natural arm as it has the feature of variable gripping force, i.e., the force applied by the hand fingers on the object is proportional to the weight of the object. To implement the feature, a relay switching system was designed which consist of relays meant for closing and opening the hand to switch different values of resistances in series with motor armature as described in author's previous work. ${ }^{8}$

Two relays are used for opening and closing limit switches and another two relays are used for implementation of grip control logic function. This function depends on the activation of particular relay switching at different time instants, according to the instructions received from microcontroller. The logic is set for three different levels of forces. Grip 1 is the lowest level logic, Grip 2 is for medium level logic and Grip 3 is for high level logic. Based on the SEMG signal exerted by the user, different resistances are selected by a relay matrix which varies the current through the motor thus varying the torque which in turn, manifests itself as grip force.

\section{Validation of the Device}

The fabrication and testing of the designed arm was carried out in the laboratory. Experimentally, the frequency components up to $40 \mathrm{~Hz}$ were found to undergo change due to muscle action. The signal was, low-pass filtered up to $100 \mathrm{~Hz}$ and amplified to increase the sensitivity. The amplitude of amplified EMG was found as $0.4-0.8 \mathrm{~V}$ in the relaxed state and $2.3-4.0 \mathrm{~V}$ during contraction in different subjects. To offset the variability of signal, a variable gain amplifier was designed, which was used to set the gain less than $1 \mathrm{~V}$ signal in relaxed state and more than $2.5 \mathrm{~V}$ during contraction. The threshold between opening and closing of hand was then set to $2 \mathrm{~V}$. The motor current during normal closing of hand was recorded as $67 \mathrm{~mA}$ which rises to more than $500 \mathrm{~mA}$ when an object is gripped. A threshold of $400 \mathrm{~mA}$ was set to cut-off the motor supply.

When the lifted object is seen by eye it gives signal to brain. In response, the brain gives the signal to arm muscles providing the information about how much heavy the object is and accordingly, the muscle contracts. This contraction is sensed by electrodes and this electromyogram signal is provided to microcontroller in digital form. The microcontroller drives the relay matrix according to the signal strength.

When power of the complete system is put $\mathrm{ON}$, the current position of hand is detected through microcontroller with the help of optical limit switches; microcontroller then restores the hand to the default 'CLOSE' position. If hand is already 'CLOSED' no action is taken by microcontroller. Surface electromyogram signal initiated by the user is consistently and continuously scanned by microcontroller and thereafter hand starts opening with predefined speed and grip force. After full opening of hand, controller again waits for close signal from the user. As user generates the close signal, the hand starts closing and grips the object with a grip force proportional to induced level of SEMG signal by the user. To implement variable grip force, current in the armature of motor is varied using a matrix of resistors with the help of relay switching. Different port bits of the microcontroller for different relay operations are set or reset as shown in Table 1.

$\mathrm{TH}_{1}, \mathrm{TH}_{2}$ and $\mathrm{TH}_{3}$ denote the progressively increasing threshold levels of SEMG signal corresponding to three different increasing grip forces exerted by hand on the object. One relay is used for the opening and closing of the hand by changing the 
Table 1. Different microcontroller port-bits function.

Grip 1: Lowest grip force

TH1 $<=$ SEMG signal $<=$ TH2

$\begin{array}{llr}\mathrm{P} 3.0=0 & \mathrm{P} 3.2=0 & \text { Motor 'ON' } \\ \mathrm{P} 3.1=0 & \mathrm{P} 3.2=1 & \text { Motor 'OFF' }\end{array}$

Grip 2: Medium grip force

TH2 $<=$ SEMG signal $<=$ TH3
P3.0 = 1 P3.3 $=0 \quad$ Direction for 'OPEN' set
$\mathrm{P} 3.1=0 \quad \mathrm{P} 3.3=1 \quad$ Direction for 'CLOSE' set
Or

$\mathrm{P} 3.0=0 \quad \mathrm{P} 3.4=0 \quad$ 'Open' limit switch reached

$\mathrm{P} 3.1=1 \quad \mathrm{P} 3.4=1 \quad$ 'Open' limit switch yet not reached

Grip 3: Highest grip force

SEMG signal $>=$ TH3

$\begin{array}{ll}\mathrm{P} 3.0=1 & \mathrm{P} 3.5=0 \\ \mathrm{P} 3.1=1 & \mathrm{P} 3.5=1 \quad \text { 'Close' limit switch reached } \\ & \end{array}$

direction of motor, while second relay is for making the motor On/Off and another two relays implement the grip force logic function. Based on the SEMG signals exerted by the user, microcontroller bits control different relays which, in turn, put different resistances in series with motor to control the current through motor thus achieving variable grip force. The complete flow chart for the working of myoelectric control based prosthetic device with following categories is shown in Fig 2.

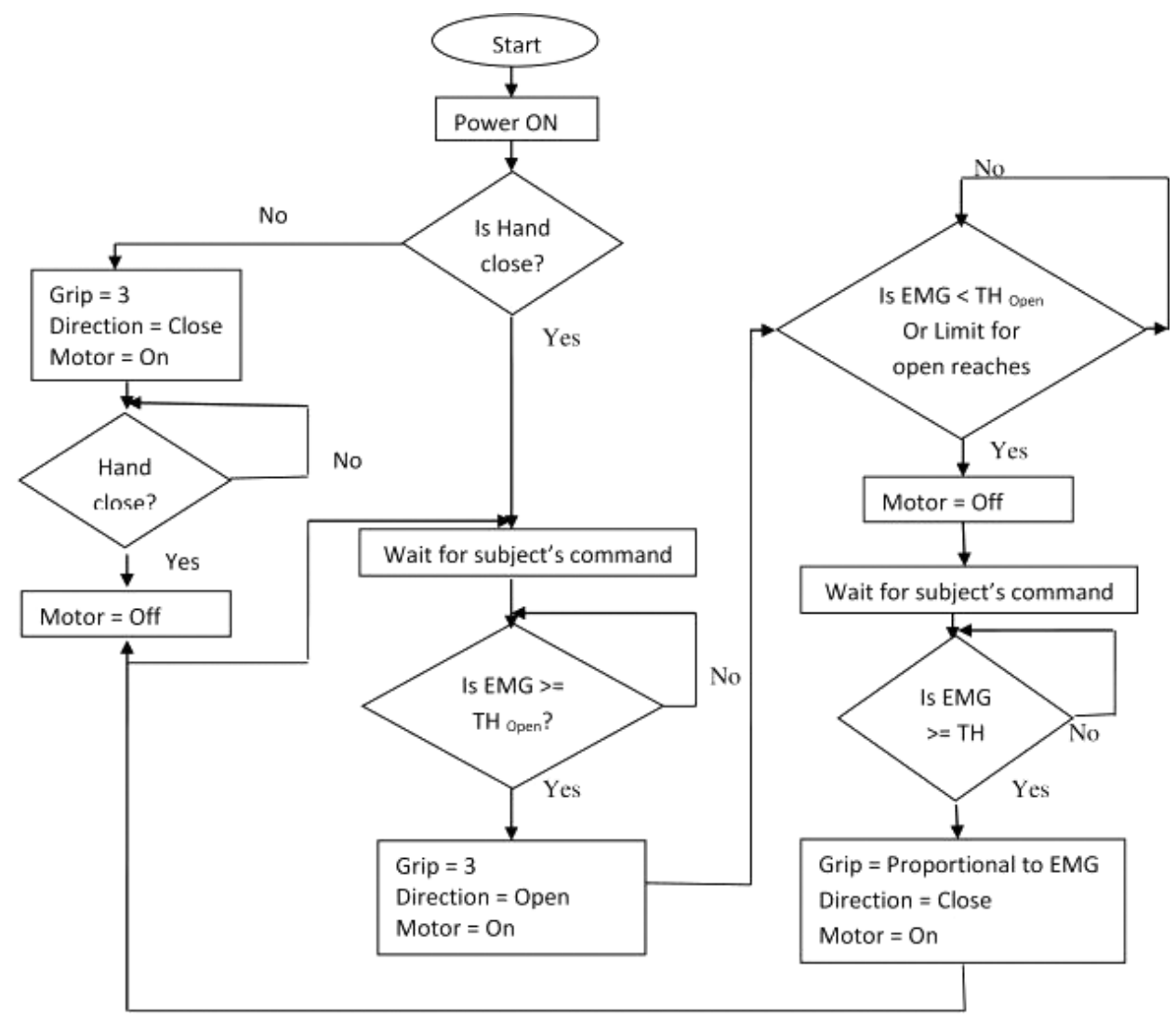

Fig. 2. Flow chart for the complete operation of myoelectric arm. 


\section{Case 1. Hand is open originally and has to close (Grip process)}

In this case, by relay switching system, direction is set for "CLOSE" position. Surface electromyogram signal has to be then exerted by subject approximately for less than $2 \mathrm{~s}$ before relaxing.

\section{Case 2. Hand is closed originally and has to open}

If the applied force is more than a particular threshold value, i.e., $\mathrm{TH}_{\text {open, }}$, it means subject is trying to open hand and then Grip 3 is set and consequently motor gets "ON" and direction of hand for "OPENING" is set.

\section{Case 3. Hand closed, it is to be opened - Releasing object}

This is just like case 1 .

The mechanical assembly of the arm consisted of wrist, palm and the fingers to grip the object in addition to a screw arrangement connected to a low power DC motor and gear assembly to open or close the hand. The wrist is mechanically rotated with the other hand to orient the artificial hand in a direction suitable to pick up the object. The entire setup is placed in a casing which provides a cosmetic appeal to the artificial hand and the connected arm.

\section{Discussion}

The one of the important component for enabling the interfacing between the nervous system and prosthesis is the electrodes "connection" to the fabricated system. Since noninvasive electrodes (planted in this current research) are required to pick up signals to stimulate muscles for feeding information back through bio-feedback system so necessary steps for amplification, processing and digitalization within the framework of these endeavors were exercised for generating the utilized SEMG signal to perform various prosthesis activities. ${ }^{17,18}$

Now, one could easily utilize these signals (S2/S4: strong contraction signal), to open or to close function and vice versa. In addition, to grasp hold function, another signal of S3 is required, if hand is in open state. Further, intelligent design protocol was carried out with two active (to open and to close) and one passive (to grasp hold) degree of freedom, respectively as shown in Fig 3. The myoelectric signal acquired through surface electrodes is more stable and helps in discriminating signal's level from each single

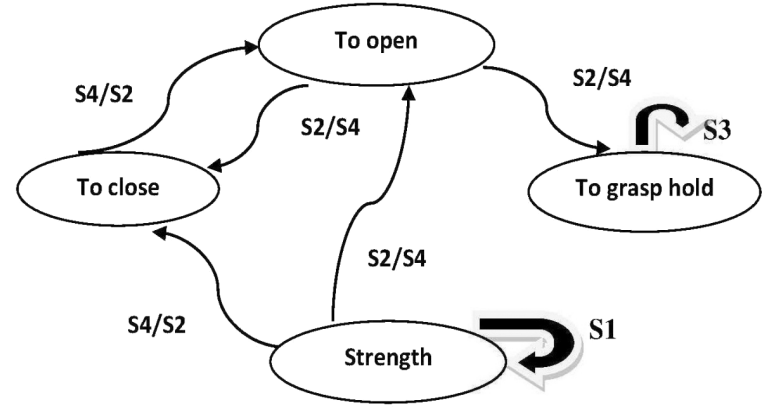

Fig. 3. The exercised activities for designed arm.

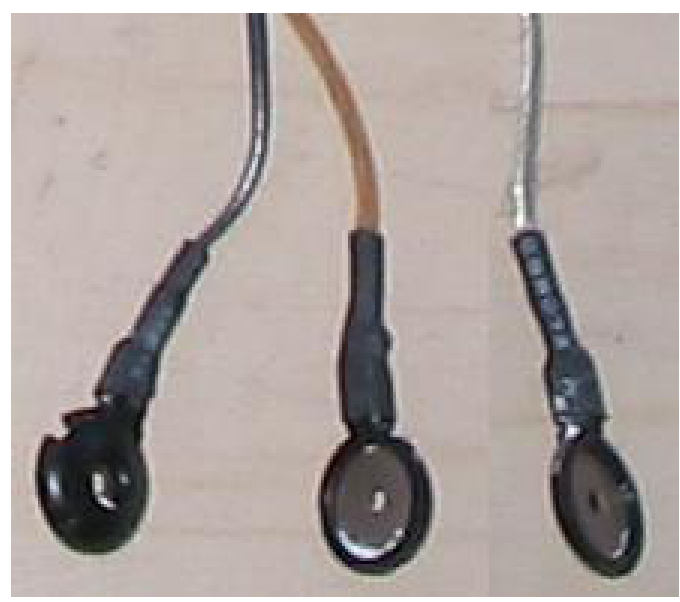

Fig. 4. The three electrodes.

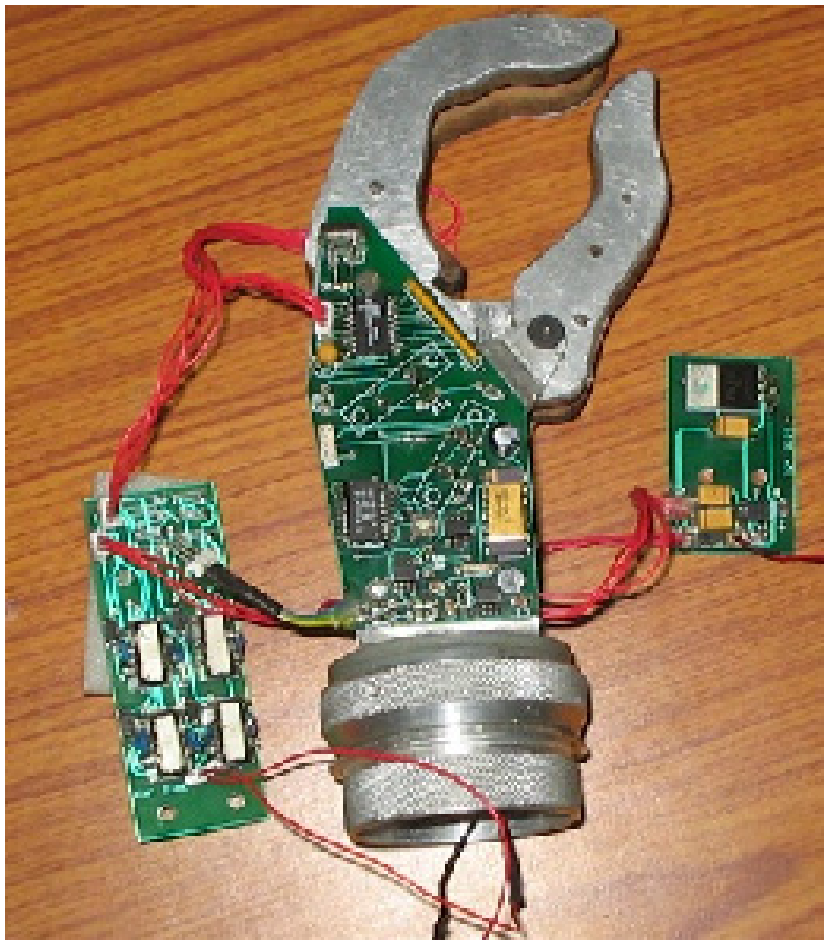

Fig. 5. Below-elbow myoelectric arm. 
Table 2. Arm specifications.

\begin{tabular}{lc}
\hline Operating voltage & $6 \mathrm{~V} / 9 \mathrm{~V}$ \\
Current consumption & $200 \mathrm{~mA}$ (Approximately) \\
Opening width & $75-100 \mathrm{~mm}$ \\
Grip force & $50 \mathrm{~N}$ (maximum) \\
Average speed & $3-4 \mathrm{~cm} / \mathrm{sec}$ (approximately) \\
Weight of arm & $1.5 \mathrm{~kg}$ (maximum) \\
On-off switch & Integrated \\
\hline
\end{tabular}

muscle and consequently, prosthesis design with three functionalities of (to open; to close and to grasp hold) could take place from the dedicated muscle movements/activities.

\section{Conclusion}

After thorough study and experimentation, biceps and triceps muscles of the residual limb were identified from which appreciable signal was obtained during voluntary contraction. Three gold plated surface electrodes were mounted above these muscles - one as reference, another as active and the third as ground electrode. For better contact between electrode and muscle, we used a conducting jelly. Experiments were made to optimally place these electrodes and to identify the proper point of axial spread of muscle where the amplitude of signal was maximal.

The electrodes (Fig. 4) cannot be placed axially along the muscles of the arm because the stump length available from such patients is not enough. The placement of this assembly in the transverse direction led to the difficulty that the surface of arm was not uniform throughout the radial span of the stump; it was rather curved. Also at the time of relaxation and contraction of the muscle, this curvature varies and the electrodes could not be fixed properly to pick the signal from a particular point.

During experiments with this assembly, it was noted that if electrodes are placed very close to each other, active and reference electrodes pick up almost the same signal from the same muscle causing no difference between them. For this reason, to get substantial signal, we placed the electrodes as far as possible from each other in the transverse direction. The complete designed below-elbow arm is as shown in Fig. 5 .

The specifications of designed prosthetic arm are as follows (Table 2):

The mechanism gave no backlash with the arm movements since it is easily controllable with the help of SEMG signals. The gear is attached to a DC motor for hand movement. Performance of whole system can be improved if jelly-less electrodes can be modified and low torque motor is replaced by high torque, higher efficiency motor, so in future active sensor can be used for carrying out the work related to control of prostheses devices.

\section{References}

1. R. B. Jerard, T. W. Williams, Practical design of an EMG controlled above elbow prosthesis, Proc. Conf. Engineering Devices for Rehabilitation, Boston, MA (1974).

2. S. George "EMG pattern analysis and classification for a prosthetic arm," IEEE Trans. Biomed. Eng. 29, 403-412 (1982).

3. C. Toledo, L. Leija, R. Muñoz, A. Vera, "Upper limb prostheses for amputations above elbow: A review," Pan American Health Care Exchange Conference, Mexico, pp. 104-108 (2009).

4. L. Chris, "Progressive upper limb prosthetics," $J$. Phys. Med. Rehabil. Clin. 17, 49-72 (2006).

5. D. Graupe, J. Magnussen, A. Beex, "A microprocessor system for multifunctional control of upper limb prosthesis via myoelectric signal identification," IEEE Trans. BME Rehabil. Eng. 2, 538-544 (1978).

6. R. Tomovic, G. Boni, "An adaptive artificial hand," IRE Trans. Automatic Control. 7, 3-10 (1962).

7. J. Stephen, "Artificial arms evolve from robots or vice versa," IEEE Control Sys. Mag. Appl. Control 25(1), 16-20 (2005).

8. K. Veer, R. Agarwal, A. Kumar, "Processing and interpretation of surface electromyogram signal to design prosthetic device," Robotica, doi: 10.1017/ S0263574714002409.

9. L. Galliano, "Research design and development project myoelectric prosthesis of upper limb, 16th Argentine Bioengineering Congress and the 5th Conf. Clinical Engineering," J. Phys. 90, 1-7 (2007).

10. J. K. Kyung, K. W. Joo, L. K. Hyun, C. B. Sung and E. H. Ki, "EMG pattern classification using spectral estimation and neural network," SICE Annual Conf., Kagawa University, Japan (2007).

11. R. Okuno, M. Yoshida, K. Akazawa, "Development of biomimetic prosthetic hand controlled by electromyogram," Advanced Motion Control MIE Proc. 4th Int. Workshop on Advanced Motion Control, Mie (1996).

12. K. Veer, R. Agarwal, "Wavelet denoising and evaluation of electromyogram signal using statistical algorithm," Int. J. Biomed. Eng. Technol. 16, 293-305 (2014). 
13. K. Veer, R. Agarwal, "Wavelet and short-time Fourier transform comparison-based analysis of myoelectric signals," J. Appl. Stati. 42, 1591-1601 (2015).

14. M. Hariharan, Y. C. Fook, R. Sindhu, I. Bukhari, Y. Sazali, "A comparative study of wavelet families for classification of wrist motions," Comput. Electri. 38, 1798-1807 (2012).

15. "Otto Bock Hand", www.ottobock.com.

16. C. A. Daniel, "Three Level innovations of the Powered above Elbow Prosthetic Arm Using Pneumatic
Artificial Muscles, M.Sc. Theses, Department of Mechanical Engineering," University of Alabama (2011).

17. K. Veer, "Interpretation of surface electromyograms to characterize arm movement," Instrum. Sci. Technol. 42, 513-521 (2014).

18. M. Silvestro, "Control of hand prostheses using peripheral information," IEEE Rev. BME. 3, 48-68 (2010). 